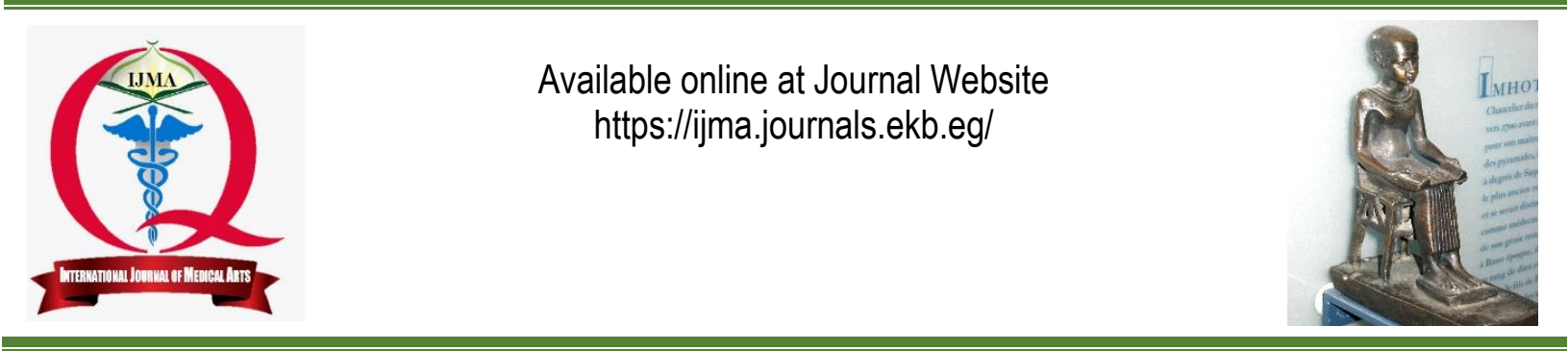

Original article

\title{
Comparison Between Unilateral and Bilateral Sacrospinous Ligament Fixation for Management of Vault Prolapse
}

\author{
Mahmoud Salah Mahmoud \\ Department of Obstetrics and Gynecology, Damietta Faculty of Medicine, Al-Azhar University, Egypt
}

Email: dr.m.s.rady@domazhermedicine.edu.eg

Received at: November 29, 2019; Revised at: January 6, 2020; Accepted at: January 6, 2020; Available online at: January 7, 2020

\section{ABSTRACT}

Background: Hysteropexy is an effective procedure for vault prolapse as abdominal sacrocolpopexy but easier and simple. Right sacrospinous fixation has the same results of bilateral fixation.

Aim of the work: To evaluate the value and safety of unilateral or bilateral sacrospinous fixation [SSF].

Patients and methods: A prospective observational study was conducted in the Department of Obstetrics and Gynecology, Al-Azhar University, Damietta over a period of two years [July 2017 to June 2019]. A total of 32 patients posted for 16 cases unilateral sacrospinous ligament fixation to vault and 16 cases for bilateral fixation were enrolled after informed consent. Results were recorded under headings of procedure time [min], blood loss [ml], major intro-operative complications and post-operative complications, postoperative pain and duration of hospital stay.

Results: The result of unilateral transvaginal sacrospinous ligament fixation technique, as part of the vaginal repair procedure for massive uterovaginal [Pelvic Organ Prolapse stage III and stage IV and vault prolapse] was evaluated as better than bilateral approach with the same efficacy, less time consuming, less blood loss and low post-operative pain.

Conclusion: Unilateral transvaginal sacrospinous ligament fixation revealed a significant reduction in intraoperative blood loss, procedure time, immediate post-operative pain, and mean length of hospital stay.

Keywords: Sacrospinous; Fixation; Ligament; Sacrocolpopexy; Unilateral.

This is an open access article under the Creative Commons license [CC BY] [https://creativecommons.org/licenses/by/2.0/]

Please cite this article as: Mahmoud MS. Surgical Comparison Between Unilateral and Bilateral Sacrospinous Ligament Fixation for Management of Vault Prolapse. IJMA 2020; 2[1]: 247-252. 


\section{INTRODUCTION}

Supporting factors for the upper part of the vagina play a vital role in surgery for pelvic organ prolapse [POP]. The process for vaginal apical support should afford a strong suspension, have few comorbidities. As much populations being older and an increasing number of females are every year experiencing pelvic organ prolapse, repair surgery that improves long-term results and reduces comorbidities is not only easy, but also required. Prior to operation, we should discuss the surgical options for POP, a normal anatomy and its variations are warranted, as anatomical variations could be responsible for the different types of POP defects. The pelvic organ system, including the uterus, urethra, bladder, vagina, rectum and perineal body, these organs are detained in position by cooperative action between pelvic bones, pelvic muscles, and fascial tissue [1].

Anterior compartment pelvic organ prolapse ascends from failing of the fascia of the levator ani muscle and descent of the bladder and urethra into the relative [potential] space of the vagina. Damage of this urethral support may lead to increased mobility of the urethra and stress urinary incontinence [SUI] without descent of the base of the bladder. Defects of bladder support may be lateral, central, or both. ${ }^{[2]}$

The incidence of vault prolapse varies from $0.2 \%$ to $38 \%$. Management options are obtainable, as pessary use, physiotherapy for pelvic floor, and surgery. The common procedures achieved for vault prolapse comprises sacrospinous ligament fixation, insertion of transvaginal mesh and abdominal sacrocolpopexy. Abdominal sacrocolpopexy had been considered for a long time as the main intervention for vaginal vault prolapse. It had been firstly described by Lane in 1957.[3]

Other Vaginal surgery procedure was used for repairing pelvic organ prolapse which support apex of the vagina includes sacrospinous ligament fixation, uterosacral ligament fixation or suspension and use of laparoscopic synthetic meshes. Abdominal approaches for vaginal apical support as sacrocolpopexy are laparotomy procedures. Although there are many other available operations, the perfect intervention for vaginal apical supporting has yet to be found. The first technique of sacrospinous ligament fixation was established and propagated by Richter. [4]

Sacrospinous ligament fixation demonstrated in Figure 1. The technique of sacrospinous ligament fixation includes a midline incision of the apex on the posterior wall of the vagina with opening of the pararectal space to reach the sacrospinous ligament. In cases who have previous surgery, sharp dissection was used. To reduce intraoperative bleeding and shorten duration of the operation, anatomical dissection should be used. When the two sutures were located with fixation at the two vaginal fornices, the vaginal wall was totally approximated. The whole maneuver usually completed within 30-50 minutes. [5]

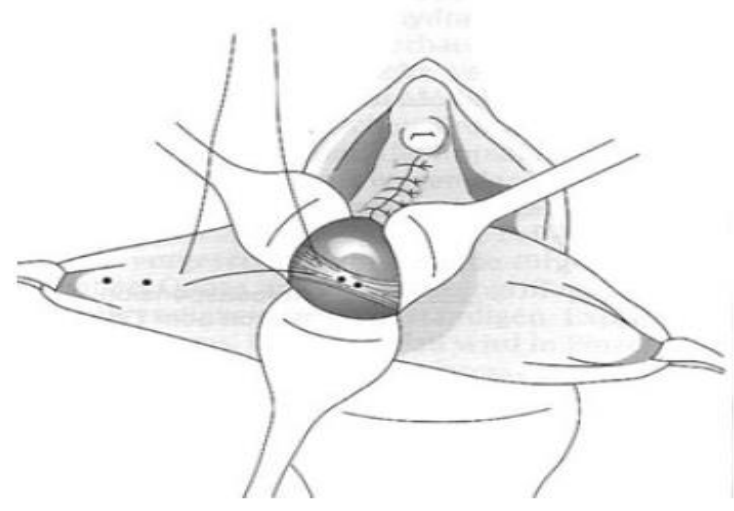

Figure [1]: Illustration of sacrospinous ligament fixation

\section{AIM OF THE WORK}

Our study aimed to evaluate the value and safety of unilateral or bilateral sacrospinous fixation [SSF].

\section{PATIENTS AND METHOD}

The study was a prospective observational study of 32 females attended Obstetrics and Gynecology Department, Al-Azhar University [Damietta] in the period between July 2017 and June 2019, who underwent bilateral sacrospinous ligament fixation. We included patients who had recurrent prolapse after previous vaginal repair. Before this procedure we use different strategies as the use of a vaginal pessary, pelvic ground physiotherapy and no response. The operation was done under local spinal anesthesia in lithotomy position for sacrospinous fixation, apical incision was carried out in the posterior vaginal wall to expose the rectovaginal area. The posterior vaginal wall was dissected bluntly laterally and the pararectal space opened 
with finger on the proper aspect. The check is most completed to the affected person's right due to the fact that, retraction of the rectum was easier and a proper-handed healthcare professional could pass a suture. We made a window among the rectovaginal area and the ischial spine by the way of the blunt dissection. The use of right finger if ischial spine diagnosed carefully, the aircraft was typically domesticated with none issue. Dissection was carried out until ischial spines were reached. We generally used the ischial backbone as a milestone, to palpate the sacrospinous ligament; this ligament passes from the ischial spine to the outer edge of the sacrum. Now, 3 slender malleable retractors were used to retract the peritoneum and rectum, to visualize definitely the sacrospinous ligament. The top border of the sacrospinous ligament was now identified. In all cases, a nonabsorbable suture monofilament polypropylene 1 , five $\mathrm{mm}$ on spherical needle forty millimeter, was used for this method, with a 12 inch strait needle holder, the suture was located through the sacrospinous ligament and coccygeus muscle beginning from the higher border of the ligament with upward to downward route, it changed into approximately $2 \mathrm{~cm}$ medial to the ischial spine, to keep away from injury of neurovascular package deal. As this suture was taken, loop shape, now it was divided to create two sutures. Thus, two suture pairs are established with one pass. The sutures were placed through the whole thickness of vaginal muscle at the socket of new vaginal apex. Vaginal cuff was now sutured and closed. The sutures of sacrospinous ligament were now ligated. This tying of sutures brings the sacrospinous ligament in direct contact with the vaginal epithelium. When healing occurred, vaginal epithelium was fixed with the sacrospinous ligament and vault remains suspended up kindly thereafter.

Postoperatively, women were given broadspectrum antibiotic for five days, who received one dose of antibiotic [As a prophylaxis] before operation.

The effects were evaluated in terms consistent with efficacy, headaches and excellence of existence after sacrospinous vaginal fixation. The efficacy became assessed by the usage of signs and symptoms of vaginal bulging, urinary and defecation signs. Intraoperative and any peri- operative complications were detected. All patients were re-examined 3 months post-operative in the outpatient clinic with evaluation of measures used before surgery.

Statistical analysis of data was accomplished by statistical package for social science [SPSS] software package, version 16 [SPSS Inc., Chicago, USA]. Arithmetic mean and standard deviations were calculated for numerical variables, while frequency and percent were calculated for qualitative parameters. Two means compared by student "t" test and qualitative data compared by Chi square test. Paired comparison carried out by paired samples "t" test or Wilcoxon singed ranks according data type and $p$ value $<0.05$ was considered significant.

\section{RESULTS}

Thirty-two females were involved in our study. Vault prolapse was after total abdominal history among 18 patients [56.25\%], two [6.25\%] after subtotal hysterectomy, 5[15.6\%] after vaginal hysterectomy and $7[21.8 \%]$ after pelvic floor repair [Table 1].

In the present work, symptoms were significantly decrease after surgery when compared to preoperative values [e.g., prolapse decreased from $71.8 \%$ to $12.5 \%$, bulge symptoms decreased from $100.0 \%$ to $9.3 \%$, stress urinary incontinence decrease from $34.4 \%$ to $12.5 \%$, overt active bladder from $25 \%$ to $3.1 \%$ [Detailed results in Table 2]. The right [unilateral] sacrospinous ligament fixation procedure was in 16 patients and bilateral sacrospinous ligament fixation in 16 patients.

When comparing unilateral to bilateral procedures, unilateral fixation was associated with older age, significant increase of BMl and longer duration of follow up [Table 3].

As regard to time consuming, unilateral approach was associated with significantly shorter time than bilateral approach [Table 4]. In addition, blood loss was significantly low with unilateral than bilateral approach [Table 5]. Finally, there was statistically significant decrease of postoperative pain score in unilateral when compared to bilateral approaches in the postoperative days 1,2 and 3 [Table 6]. 
Mahmoud MS.

Table [1]: Vault prolapse following surgery

\begin{tabular}{|r|r}
\hline \multicolumn{1}{|c|}{ Pervious surgery } & Value \\
\hline Total abdominal hysterectomy [TAH] & $18[56.25]$ \\
Subtotal hysterectomy & $2[6.25]$ \\
Vaginal hysterectomy & $5[15.6]$ \\
Pelvic floor repair & $7[21.8]$
\end{tabular}

Table [2]: Preoperative Symptoms and symptoms at 3 months postoperatively [no=32]

\begin{tabular}{|l|c|c|}
\hline \multicolumn{1}{|c|}{ Outcome } & Preoperative & Symptoms after surgery \\
\hline Prolapse & $23[71.8]$ & $4[12.5]^{*}$ \\
Bulge symptoms & $32[100]$ & $3[9.3]^{*}$ \\
Stress urinary incontinence & $11[34.4]$ & $4[12.5]^{*}$ \\
Over active bladder & $8[25]$ & $1[3.1]^{*}$ \\
Bowel dysfunction & $2[6.25]$ & $2[6.25]$ \\
Sexual dysfunction & $11[34.3]$ & $3[9.3]^{*}$ \\
\hline
\end{tabular}

* significant reduction after surgery

Table [3]: Comparison between studied groups regarding patient characters

\begin{tabular}{|l|c|c|c|}
\hline & USSHP & BSSHP & P value \\
\hline Age & $56.6 \pm 10.4$ & $48.2 \pm 6.2$ & $0.001^{*}$ \\
\hline Parity & $7 \pm 3.1$ & $6.2 \pm 2.9$ & 0.304 \\
\hline BMl & $29.7 \pm 5.4$ & $29.5 \pm 3.6$ & $0.001^{*}$ \\
\hline Discharge & $3.7 \pm 2.3$ & $3.1 \pm 3.0$ & 0.426 \\
\hline Follow up & $25.5 \pm 26.3$ & $13.9 \pm 8.1$ & $0.043^{*}$ \\
\hline
\end{tabular}

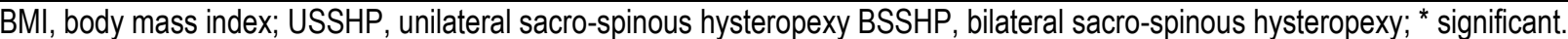

Table [4]: Time consuming for unilateral group versus bilateral group

\begin{tabular}{|c|c|c|c|c|c|}
\hline \multirow[t]{2}{*}{ Time interval } & \multicolumn{2}{|c|}{ USSH } & \multicolumn{2}{|c|}{ BSSH } & \multirow[t]{2}{*}{ P value } \\
\hline & n. & $\%$ & $\mathrm{n}$. & $\%$ & \\
\hline 20-30 min & 9 & 56.3 & 0 & 0 & \multirow{7}{*}{$<0.001^{*}$} \\
\hline $30-40 \mathrm{~min}$ & 5 & 31.3 & 0 & 0 & \\
\hline $40-50 \mathrm{~min}$ & 2 & 12.5 & 5 & 31.3 & \\
\hline $50-60 \mathrm{~min}$ & 0 & 0 & 5 & 31.3 & \\
\hline $60-70 \mathrm{~min}$ & 0 & 0 & 3 & 18.8 & \\
\hline $70-80 \mathrm{~min}$ & 0 & 0 & 2 & 12.5 & \\
\hline $80-90 \mathrm{~min}$ & 0 & 0 & 1 & 6.5 & \\
\hline
\end{tabular}

* Significant

Table [5] Blood loss for unilateral group versus bilateral group

\begin{tabular}{|c|c|c|c|c|c|}
\hline \multirow[t]{2}{*}{ Volume } & \multicolumn{2}{|c|}{ USSH } & \multicolumn{2}{|c|}{ BSSH } & \multirow[t]{2}{*}{$P$ value } \\
\hline & n. & $\%$ & $\mathrm{n}$. & $\%$ & \\
\hline $120-140 \mathrm{ml}$ & 10 & 31.25 & 2 & 9.37 & \multirow{5}{*}{$<0.001^{*}$} \\
\hline $140-160 \mathrm{ml}$ & 3 & 6.25 & 1 & 3.12 & \\
\hline $160-180 \mathrm{ml}$ & 2 & 9.37 & 2 & 9.37 & \\
\hline $180-200 \mathrm{ml}$ & 1 & 3.12 & 3 & 6.25 & \\
\hline $200-220 \mathrm{ml}$ & 0 & 0 & 8 & 25 & \\
\hline
\end{tabular}

USSH unilateral sacro-spinous Hysteropexy, BSSH bilateral sacro-spinous hysteropexy, ${ }^{*}$ significant

Table [6]: Postoperative pain in relation to surgical approach

\begin{tabular}{|l|c|c|}
\hline \multicolumn{1}{|c|}{ Postoperative pain } & USSH & BSSH \\
\hline Post-op day 1 & $6.15^{*}$ & 8.44 \\
\hline Day 2 & $3.48^{*}$ & 5.31 \\
\hline Day 3 & $1.090^{*}$ & 2.8422 \\
\hline
\end{tabular}

* significant decrease in USSH versus BSSH 


\section{DISCUSSION}

The present trial assessed each anatomical and efficient outcomes of an original procedure of bilateral sacrospinous fixation versus unilateral sacrospinous fixation to help support and management of vault prolapse. The technique offered an excellent support for vault prolapse in $90 \%$ of patients who doesn't underwent vaginal operation of prolapse, and suspended the vaginal vault. Three patients had a mild cystocele at postoperative 3 months follow-up and most effective one among them wanted a consequent repair. In comparison to previous study, similar outcomes were noted by Khurana et al.[6], who advocated the bilateral sacrocolpopexy as an effective choice for apical prolapse.

The encountered intraoperative complication in the current work was minimal. Two [6.25\%] females had minor wound-related comorbidities in the direct postoperative period, as in previous work ${ }^{[4]}$.

The findings of this study take a look and proposed that, sacrospinous ligament fixation appears to be associated with a great efficacy, less complication, short period and much less blood loss in contrast to abdominal sacrocolpopexy and there may be no foremost distinction in unilateral or bilateral sacrospinous ligament fixation either achievement or recurrence. [5] The sacrospinous fixation may additionally decrease operative time, blood loss and time needed for postoperative recovery, and these results agree with Persu et al. ${ }^{[5]}$

In another study, BMl and parity were comparable between groups and the procedure outcome did not affected by any of BMI or parity, regarding anatomical attainment or prolapse recurrence ${ }^{[7]}$, which in contradiction to results of the present work, and could be explained by different inclusion criteria and sample size.

The present work depicted significant reduction of the operative time. The intra-operative procedure time was noted beginning from initial incision on vaginal mucosa till whole fixation to sacrospinous ligament. This close point was deliberately chosen to exclude the procedure time for cystocoele, enterocoele and rectocoele repair and other concomitant procedures that were done according to the needs of patients, as reported in previous work[5]. Most studies, indeed, depicted reduction in intra-operative time, one showed reduction of 61 to $30 \mathrm{~min}$ and the other reported operative time to be $[40 \pm 8$ min versus $65 \pm 10$ minutes in unilateral versus bilateral approaches respectively] ${ }^{[4,8]}$. In a few studies, however, the difference did not reach significance in terms of intra-operative time such as those of Lakeman et al.[9] [71.3 min versus $59.7 \mathrm{~min}$ ] and Hefni and El-Toukhy ${ }^{[10]}$ [57 versus 66 minutes].

Sacrospinous ligament fixation is a good technique in supporting vaginal vault prolapse. But, recurrent prolapse of the vault after sacrospinous ligament fixation were described. There are many factors responsible for the recurrence after sacrospinous ligament fixation. These include weakness of the supporting ligament as a result of aging and menopause, congenital weakness of supporting structures, inadequate repair with avulsion of sutures from vaginal supporting tissues [11-13].

We advocated mesh interposition and reinforcement for sacrospinous ligament fixation. The operative time is reasonable, with effective apical support, minimal complications and unified surgical technique. The supporting mesh with sacrospinous ligament fixation through vaginal approach is the procedure of choice for recurrent prolapse after failed apical repair. Restating sacrospinous ligament vaginal vault fixation is a difficult procedure, especially with scaring pararectal space and distortion of anatomical plans with increased the risk of rectal injury. An alternative approach must do sacrospinous ligament fixation at contralateral side. This is supported by previous studies[8,14-16].

Barber et al. [17] reported significant reduction of pain after surgical correction surgery after vaginal vault prolapse, with no significant difference between surgical procedures.

We conducted present study on a mixed group of patients with various indications, with different surgical difficulties in different groups. Inferences of each indication could not be drawn. Other limitations were small sample size and lack of randomization, as done in other studies. In order to give more valuable results, further research is needed with larger sample size. 
In conclusion, unilateral sacrospinous fixation is an effective alternative to conventional suturing. Unilateral sacrospinous ligament fixation is effective and significantly reduces intra-operative blood loss, procedure time, and immediate post-operative pain. However, we found that there is no a significant difference in intra-operative complications as bladder injury or bowel perforation. None of the patients in our study needed conversion to laparotomy or readmission for any major bleeding.

\section{Financial and Conflict of interest disclosure}

Authors declare that there was no conflict of interest.

\section{REFERENCES}

1. Stahel PF, Hammerberg EM. History of pelvic fracture management - a review. World J Emerg Surg. 2016; 11:8. [DOI:10.1186/s13017-016-00754].

2. Papakostidis C, Giannoudis PV. Pelvic ring injuries with haemodynamic instability: efficacy of pelvic packing, a systematic review. Injury. 2009; 40[4]: 53-61. [DOl: 10.1016/ j. injury.2009.10.037].

3. Schmal H, Markmiller M, Mehlhorn AT, Sudkamp NP. Epidemiology and outcome of complex pelvic injury. Acta Orthop Belg. 2005; 71[1]:41-7. [PMID:15792206].

4. Halawi MJ. Pelvic ring injuries: Emergency assessment and Management. J Clin Orthop Trauma 2015; 6: 252-8. [DOI: 10.1016/ j.jcot. 2015.08.002].

5. Hak DJ, Smith WR, Suzuki T. Management of hemorrhage in life threatening pelvic fracture. J Am Acad Orthop Surg. 2009; 17:447-57. [DOI: 10.5435/ 00124635-200907000-00005].

6. Khurana B, Sheehan SE, Sodickson AD, Weaver MJ. Pelvic ring fractures: what the orthopedic surgeon wants to know. Radiographics. 2014; 34[5]:1317-33. [DOI: 10.1148/rg.345135113].
7.Trainham L, Rizzolo D, Diwan A, Lucas $T$. Emergency management of high-energy pelvic trauma. JAAPA. 2015; 28:28-33. [DOI: 10.1097/01. JAA. 0000473359. 00222.a0].

8.Galluzzo M, Gaudino F, Addeo G. Pelvic Trauma: Bone. Chapter 20, in: V. Miele, M. Trinci [eds.], Diagnostic Imaging in Polytrauma Patients, Springer International Publishing AG .2018; 7:43350.

9. Woodley SJ, Kennedy E, Mercer SR. Anatomy in practice: the sacrotuberous ligament. $\mathrm{N} \mathrm{Z} \mathrm{J}$ Physiother. 2005; 33: 91-4.

10. Wan Faisham WI, Nawaz AH Joehaimey J, Sallehuddin AY, Wan Z. Anterior stabilization of sacroiliac joint for complex pelvic injuries. Malays $\mathrm{J}$ Med Sci. 2009; 16:47-51. [PMID: 22589666].

11. Suzuki T, Hak DJ, Ziran BH, Adam AS, Stahel PF, Smith RW. Outcome and complications of posterior transiliac plating for vertical unstable sacral fracture. Injury 2009; 40:405-9. DOI:10.1016/j.injury. 2008. 06.039 .

12. Choy WS, Kim KJ, Lee SK, Park HJ. Anterior Pelvic Plating and Sacroiliac Joint Fixation in Unstable Pelvic Ring Injuries. Yonsei Med J. 2012; 53 [2]: 422-6.

13. Elmanawy M, Elshoura S, Youssef S, Salama F. Treatment of sacroiliac joint disruption with anterior stabilization. Egypt Orthop J. 2015; 4:45-51. [DOI: 10.4103/ 1110-1148.163148].

14. Sagi HC, Militano U, Caron T, Lindvall E. A comprehensive analysis with minimum 1-year follow-up of vertically unstable transforaminal sacral fractures treated with triangular osteosynthesis. J Orthop Trauma. 2009; 23[5]:313-9. [DOI:10.1097/ BOT.0b013e 3181 a32b91].

15. Pohlemann T, Culemann U, Tosounidis G, Kristen A. [Application of the pelvic C-clamp]. Unfallchirurg. 2004; 107[12]:1185-91. [English abstract] [DOI:10.1007/ s00113-004-0896-6] 